\title{
Une brève histoire du climat de la Terre
}

Gilles Ramstein (gilles.ramstein@lsce.ipsl.fr)

Laboratoire des Sciences du Climat et de l'Environnement (UMR 8212 CEA-CNRSUVSQ/IPSL), Orme des Merisiers, Bât. 701, CEA/Saclay, 91191 Gif-sur-Yvette Cedex

L'histoire du climat de la Terre est un écheveau de processus

physiques dominants

à différentes échelles de temps, mais toujours imbriqués.

On peut représenter cette évolution comme une valse à quatre temps. Celui du milliard d'années,

où c'est essentiellement l'évolution de la luminosité du Soleil qui

l'emporte. Puis, à l'échelle

de dizaines de millions d'années, c'est la tectonique des plaques

qui, par son effet sur le climat

et sur la teneur en $\mathrm{CO}_{2}$

de l'atmosphére, va jouer un rôle

prépondérant. Le troisième temps

est celui de la variation

des paramètres orbitaux

à des périodes de la dizaine

à la centaine de milliers d'années.

Enfin, le quatrième et ultime

temps est celui de l'Homme qui,

en quelques centaines d'années,

s'est hissé au rang d'acteur

majeur du changement climatique.
L'histoire des climats de la Terre est d'autant plus difficile à reconstituer que l'on s'éloigne dans le temps. Nous allons néanmoins plonger dans l'histoire climatique de notre petite planète jusqu'à il y a environ 4 milliards d'années (4 Ga).

On prétend souvent que l'histoire de la Terre, de Mars et de Vénus a été semblable dans un lointain passé. En tout état de cause, le climat actuel de nos deux voisines est très différent du nôtre. Mars ne dispose que d'une atmosphère ténue en dioxyde de carbone $(\sim 600 \mathrm{~Pa})$, il y fait froid $\left(-63{ }^{\circ} \mathrm{C}\right.$ en moyenne, avec un fort cycle diurne). Vénus, au contraire, est entourée d'une atmosphère dense où les gaz à effet de serre produisent à sa surface une température de fournaise $\left(460{ }^{\circ} \mathrm{C}\right.$ en moyenne).

Sur Terre, ce qui est remarquable et un peu énigmatique, c'est que notre planète ait pu traverser plus de 4 milliards d'années en gardant à sa surface un cycle hydrologique et des températures compatibles avec la vie. Un des premiers scientifiques à conceptualiser cette régulation est James Lovelock connu pour sa théorie "Gaia » où la Terre, considérée comme un être vivant, s'adaptait à différents types d'agressions [1].
Ses conceptions, bien que très controversées ${ }^{(a)}$, apportèrent beaucoup sur les aspects biologiques, compétitions d'espèces et rétroactions du système Terre. Pour les physiciens des sciences de la Terre et de l'atmosphère, il fallait trouver des mécanismes qui permettent à notre planète de réguler sa température de surface. À travers ce voyage dans le passé de la Terre, nous allons montrer comment ces régulations sont imbriquées aux différentes échelles de temps : du passé très lointain jusqu'à la perturbation globale que nous, les Hommes, faisons subir à notre planète et que l'on appelle la perturbation anthropique.

De manière forcément pointilliste, nous reviendrons sur quelques épisodes-clés de l'histoire des climats de la Terre, et nous montrerons que l'expérience de l'Anthropocène est à la fois inédite par sa rapidité, mais également par le contexte dans lequel elle intervient.

Pour voyager à travers les climats de la Terre, il faut se munir de cartes temporelles qui vont vous permettre de prendre vos marques dans l'histoire géologique de notre planète (fig. 1).

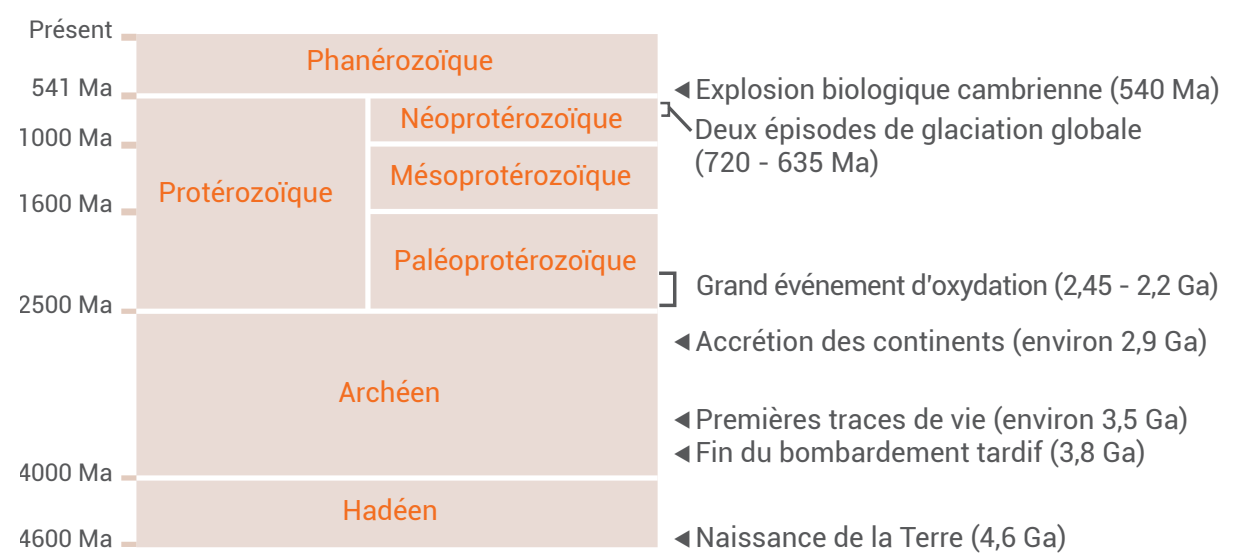

1. Les différentes périodes de l'histoire géologique de la Terre.

(a) Vue globale, de la naissance de la Terre jusqu'à l'époque présente. 

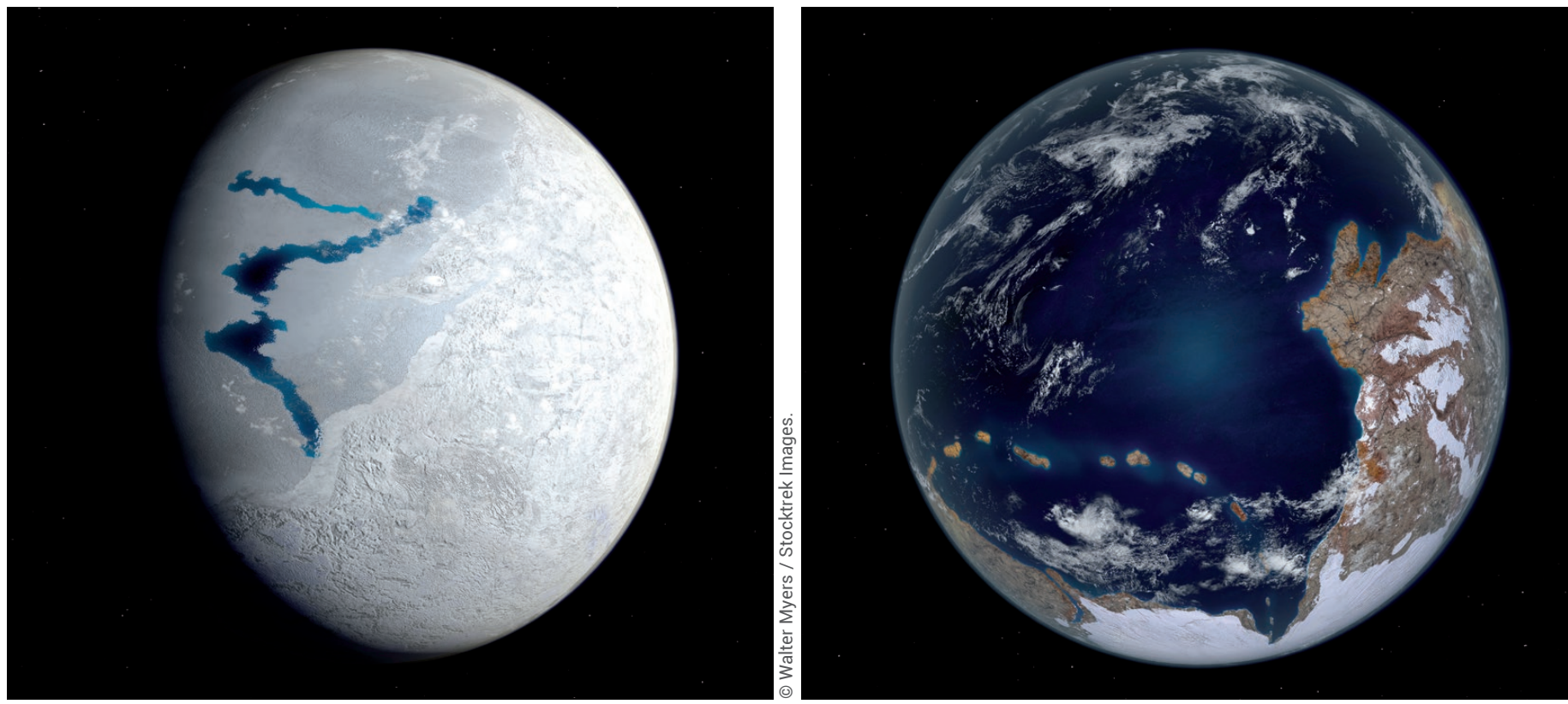

Vues de la Terre.

À gauche : il y a $650 \mathrm{Ma}$, durant la glaciation marinoenne ("Terre boule de neige ").

À droite : il y a 600 Ma, à l'issue des glaciations du Néoprotérozoïque.

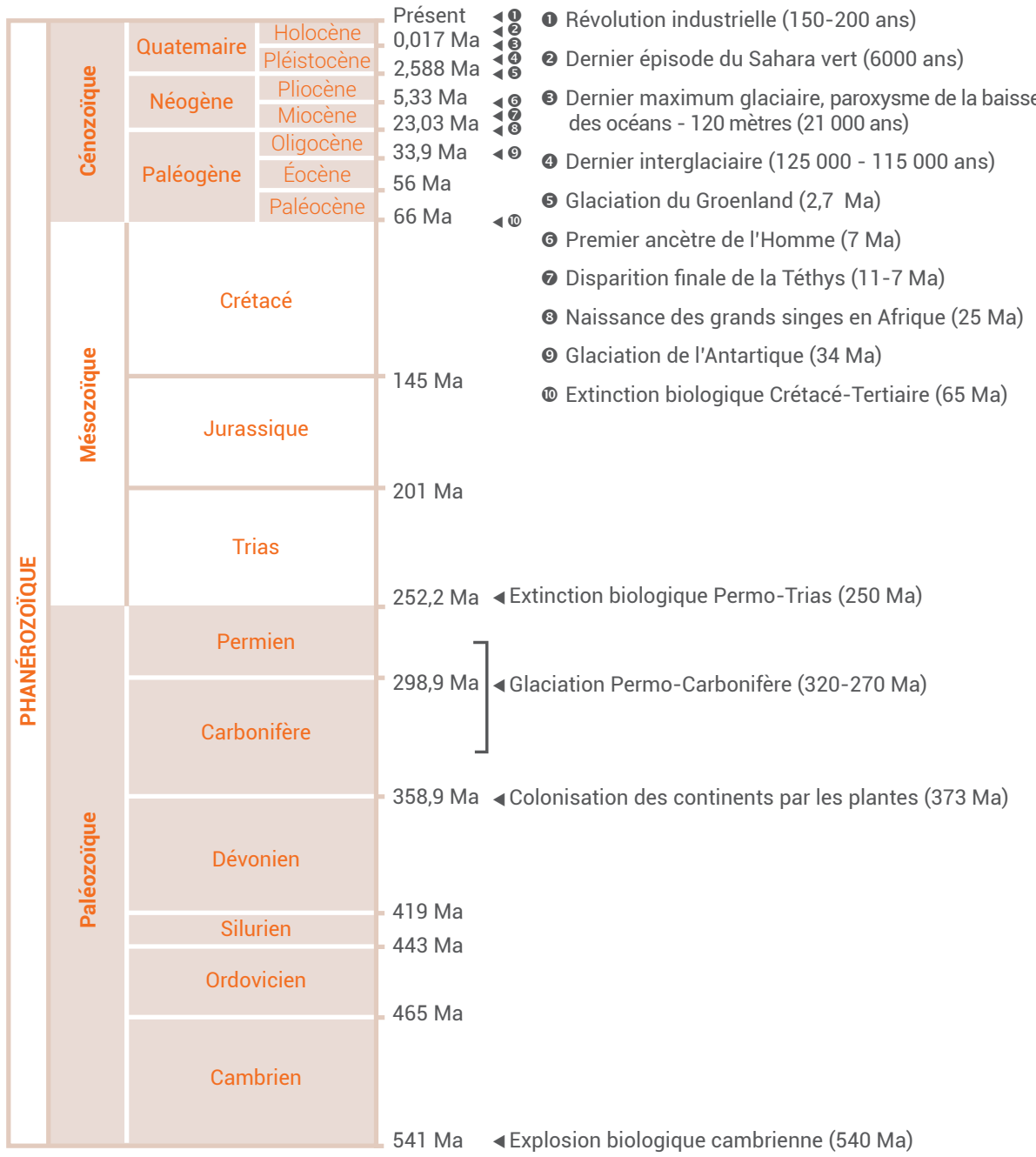

(b) Repères chronologiques simplifiés du Phanérozoïque (540 derniers millions d'années). (c) Nada Caud (LSCE), d'après la charte internationale stratigraphique.

\section{Le paradoxe du Soleil jeune}

L'une des premières surprises est que nous soyons là, sur notre planète bleue, dans le système solaire, alors que, toutes choses égales par ailleurs, sa surface devrait plutôt ressembler à ces gros satellites gelés que sont Encelade (satellite de Saturne) et Europe (satellite de Jupiter).

En effet, plus de $99 \%$ de l'énergie qui arrive à la surface de la Terre provient du Soleil. Or, ce dernier n'est qu'un réacteur nucléaire qui brûle son hydrogène et le transforme en hélium. Un avantage de notre Soleil est qu'il est une étoile tout à fait ordinaire, et qu'il existe des milliards d'étoiles identiques dans notre galaxie. On connait donc parfaitement son évolution et on peut calculer très exactement l'intensité du rayonnement solaire qui arrive au sommet de l'atmosphère terrestre au cours du temps. Cette intensité augmente d'environ 7\% par milliard d'années (fig. 2).

Ainsi, lors de la formation de notre système planétaire (il y a 4,6 Ga), la jeune Terre ne recevait du Soleil que $70 \%$ de ce qu'elle reçoit aujourd'hui. Tous les modèles radiatifs montrent que dans de telles conditions et toutes choses égales par ailleurs, la Terre aurait dû s'englacer complètement et rester des milliards d'années dans cet état. En effet, pour déglacer une Terre gelée, le Soleil aurait dû voir sa luminosité augmenter d'au moins un facteur 1,5 ce qui aurait, 
Luminosité solaire normalisée

à la valeur actuelle

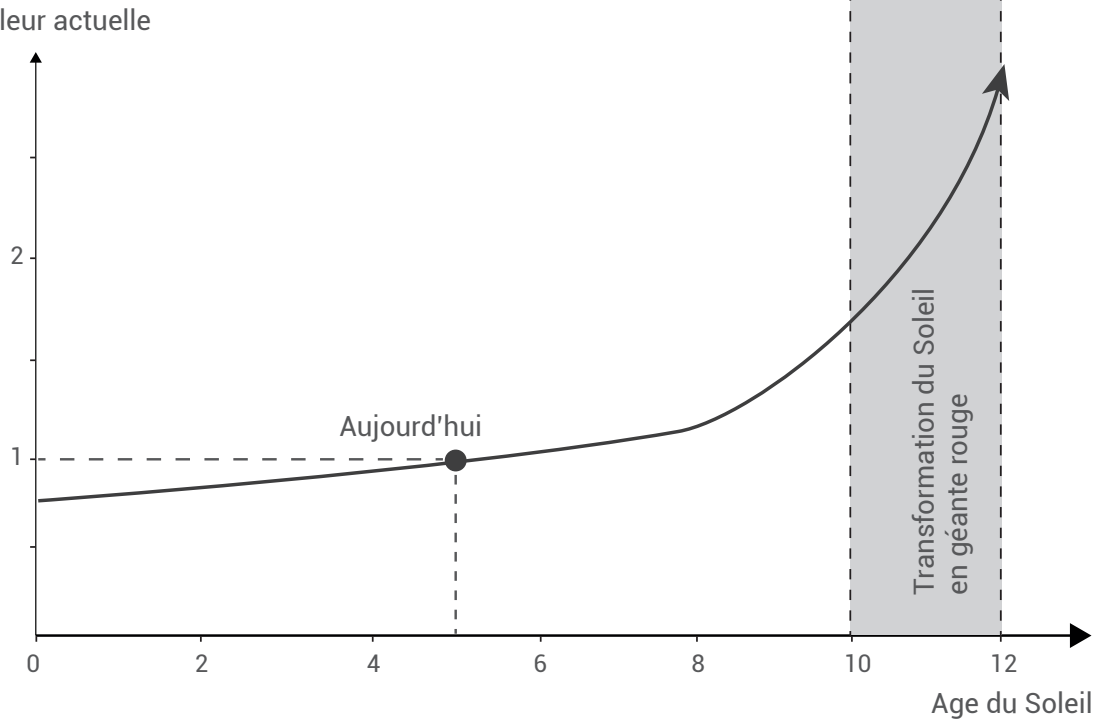

(en milliards d'années)

2. Évolution de l'intensité lumineuse du Soleil. Dans sa séquence principale, avant que le Soleil se transforme en géante rouge à un âge d'environ 10 à $12 \mathrm{Ga}$, son intensité lumineuse augmente presque linéairement avec le temps, de $7 \%$ par milliard d'années dans cette phase. (D'après D.O. Gough, University of Cambridge, U.K.).

Le cycle exosphérique du carbone à l'échelle du million d'années

Ce cycle est décrit sur la figure ci-dessous. Seuls les flux géologiques sont pris en compte, tous les recyclages rapides (flux biosphériques et interface océan-atmosphère) sont négligés. Seul le bilan de l'érosion silicatée consomme une molécule de $\mathrm{CO}_{2}$ atmosphérique.

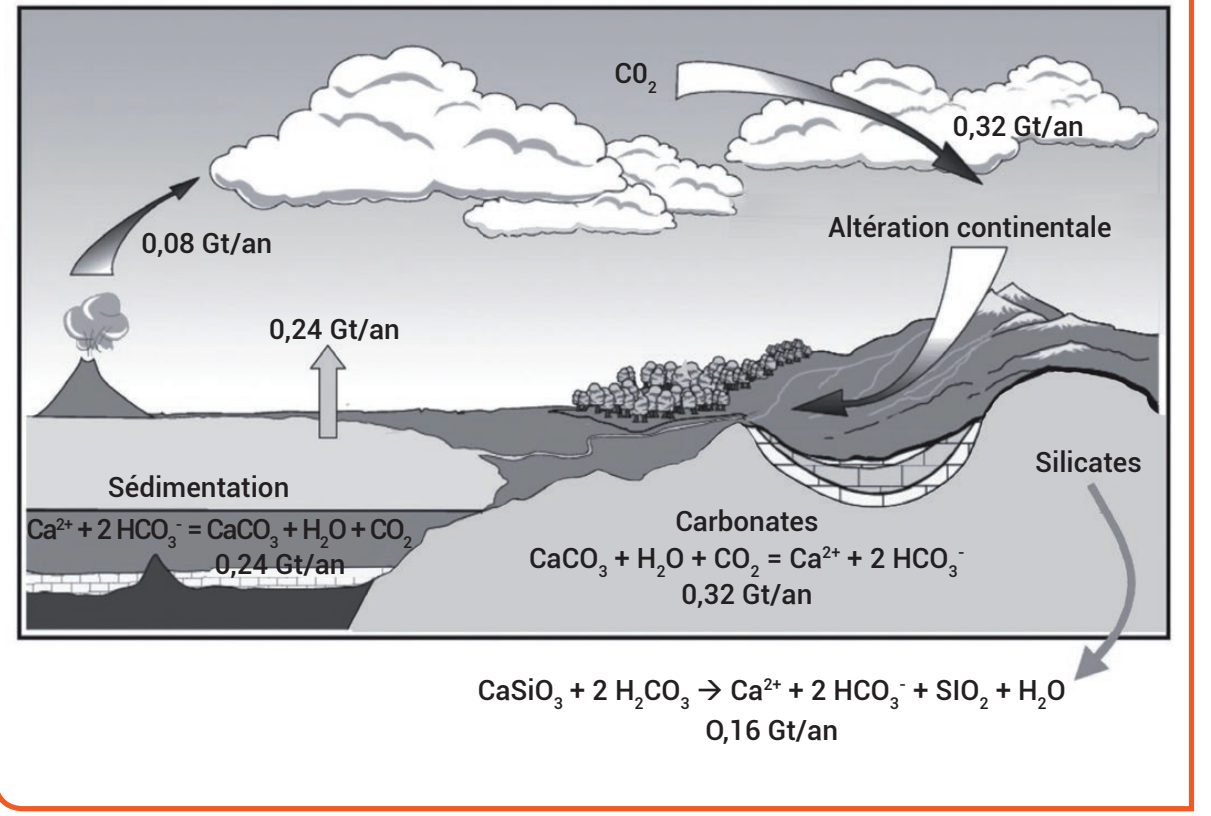

$\gg>$

comme on peut le constater sur la figure 2, pris des milliards d'années. Or, toutes sortes de données prouvent que non seulement la surface de la Terre n'a pas été englacée, mais en plus qu'elle était chaude. On peut en effet reconstituer une température des océans à partir des isotopes de l'oxygène et $\mathrm{du}$ silicium. Ces températures étaient d'environ $50{ }^{\circ} \mathrm{C}$ à l'Archéen et de $30^{\circ} \mathrm{C}$ au Protérozoïque, même si de nouvelles estimations convergent vers des températures plus basses.

Il y a donc là une énigme : avec un Soleil jeune et moins puissant, la Terre, au lieu de s'englacer, portait déjà des océans chauds. C'est ce qu'on appelle le paradoxe du Soleil jeune. Très vite, les physiciens pointent du doigt les gaz à effet de serre comme responsables des températures chaudes maintenues à la surface de la Terre dans ce contexte thermiquement défavorable. Carl Sagan a d'abord émis l'hypothèse que le responsable pouvait être l'ammoniac $\mathrm{NH}_{3}$. En effet, c'est un gaz à effet de serre très puissant, mais il est détruit par photolyse au sommet de l'atmosphère et son taux d'équilibre dans l'atmosphère restait trop faible pour protéger la Terre de l'englacement pendant les premiers milliards d'années.

Après quelques tâtonnements, le suspect le mieux placé s'avérait être le gaz carbonique qui est aussi un gaz à effet de serre. Le dioxyde de carbone possède les propriétés physiques idéales pour s'équilibrer dans l'atmosphère de la jeune Terre (pendant au moins les deux premiers milliards d'années) à un fort taux. Examinons les sources et les puits du $\mathrm{CO}_{2}$ atmosphérique à l'échelle géologique, afin de quantifier à quelle valeur il peut s'équilibrer. Les sources d'abord : elle sont essentiellement liées au volcanisme. Nous pouvons raisonnablement penser que l'intérieur de la Terre étant plus chaud qu'actuellement, le volcanisme devait être plus important. Mais c'est surtout les puits de $\mathrm{CO}_{2}$ atmosphérique qui vont nous intéresser. Ce qui permet d'extraire du $\mathrm{CO}_{2}$ de l'atmosphère, c'est l'érosion des silicates. Comme illustré dans l'encadré ci-contre, les ions $\mathrm{Ca}^{2+}$ et $\mathrm{HCO}_{3}{ }^{-}$sont emmenés à l'océan par les rivières. Si l'océan est saturé par rapport aux minéraux carbonatés, cet apport d'alcalinité va provoquer la précipitation de carbonates de calcium, et donc le stockage d'un carbone exosphérique dans l'enveloppe sédimentaire de la Terre. Mais en l'absence de continents, pas d'érosion 
silicatée, et le carbone s'accumule dans l'atmosphère. Nous pouvons considérer que jusqu'à il y a 3 milliards d'années, date à partir de laquelle l'accrétion continentale devient significative, le principal puits de $\mathrm{CO}_{2}$ ne fonctionnait pas, et que la concentration de $\mathrm{CO}_{2}$ dans l'atmosphère a pu atteindre des valeurs suffisamment importantes pour que l'effet de serre puisse compenser la plus faible luminosité d'un Soleil jeune. Il y a néanmoins un grain de sable qui vient compliquer ce scénario.

Dès qu'on a pu analyser les sols des vieux cratons $^{(b)}$ (datant de 2,5 à 3,2 Ga), on a constaté qu'il n'y avait dans ces sols nulle trace de sidérite $\left(\mathrm{FeCO}_{3}\right)$. L'atmosphère devait donc contenir moins de 0,015 bar de $\mathrm{CO}_{2}$, sinon la production de sidérites aurait dû se produire. Mais avec un tel taux de $\mathrm{CO}_{2}$, l'effet de serre n'était pas assez puissant pour éviter une glaciation globale.

Dans ce contexte, il lui a fallu un comparse. Le méthane $\left(\mathrm{CH}_{4}\right)$ n'a, a priori, pas le profil. Du point de vue radiatif, il est certes un candidat sérieux ( 25 à 30 fois plus efficace que le $\mathrm{CO}_{2}$ ), mais il rencontre deux problèmes pour s'établir à une haute teneur dans l'atmosphère. D'une part, son temps de résidence est bien plus court que celui du $\mathrm{CO}_{2}$, à cause du puits que constitue la réaction d'oxydation $\mathrm{CH}_{4}+2 \mathrm{O}_{2} \rightarrow \mathrm{CO}_{2}+2 \mathrm{H}_{2} \mathrm{O}$. Ainsi, la durée de vie du méthane est de dix ans dans l'atmosphère, dans les conditions actuelles. D'autre part, sur Terre contrairement à Titan, le plus gros satellite de Saturne, la production de méthane n'a pu être que biologique. Sur Titan, l'atmosphère est très riche, le méthane en est une composante importante, mais Titan est situé très loin du Soleil dans un tout autre contexte qui rend possible la formation de méthane de manière non biologique. Mais ces deux problèmes n'ont peut-être pas empêché d'atteindre des taux importants de méthane dans l'atmosphère des deux premiers milliards d'années de la Terre. En effet, il ne faut pas oublier que jusqu'au GEO (Grand Événement d'Oxydation) il y a 2,4 Ga, il n'y a pas d'oxygène dans l'atmosphère. Par conséquent, le puits de méthane est très affaibli. Le problème de la source est résolu par l'hypothèse de J. Kasting (professeur à l'Université de Penn State), qui s'appuie sur le fait que les archées méthanogènes ${ }^{(c)}$ qui apparaissent très tôt $(3,5 \mathrm{Ga})$ ont pu produire du $\gg\rangle$ méthane en abondance, du moins jusqu'au GEO puisque l'oxygène, même à faibles doses, est un poison pour elles.

Ces deux gaz à effet de serre, dioxyde de carbone et méthane, sont restés pendant des milliards d'années à des taux suffisants dans l'atmosphère terrestre évitant ainsi toute glaciation, malgré un Soleil jeune moins puissant... sauf à deux occasions. On ne dispose en effet que de deux épisodes de dérégulation massive où la Terre a pu s'englacer totalement. Même si sur des périodes aussi éloignées l'absence de preuve de glaciation ne représente pas forcément la preuve de leur absence, il reste néanmoins que cette régulation semble avoir fonctionné pendant des milliards d'années.

\section{Des glaciations globales Première glaciation et grand événement d'oxydation (GEO)}

La première grande glaciation est concomitante au GEO où la Terre a connu une glaciation massive, liée à l'effondrement du méthane lors de l'irruption de l'oxygène dans l'atmosphère terrestre il y a 2,45 Ga (fig. 3). La seconde est beaucoup plus récente : elle se situe au Néoprotérozoïque (entre $1 \mathrm{Ga}$ et $540 \mathrm{Ma}$ ), lorsque le $\mathrm{CO}_{2}$ s'effondre à son tour (fig. 3).

Il est extrêmement difficile de modéliser le climat d'il y a 2,4 Ga correspondant à la première glaciation, car on ne connait pas assez bien la distribution des continents. En effet, le paléomagnétisme permet de décrire l'évolution des continents et des

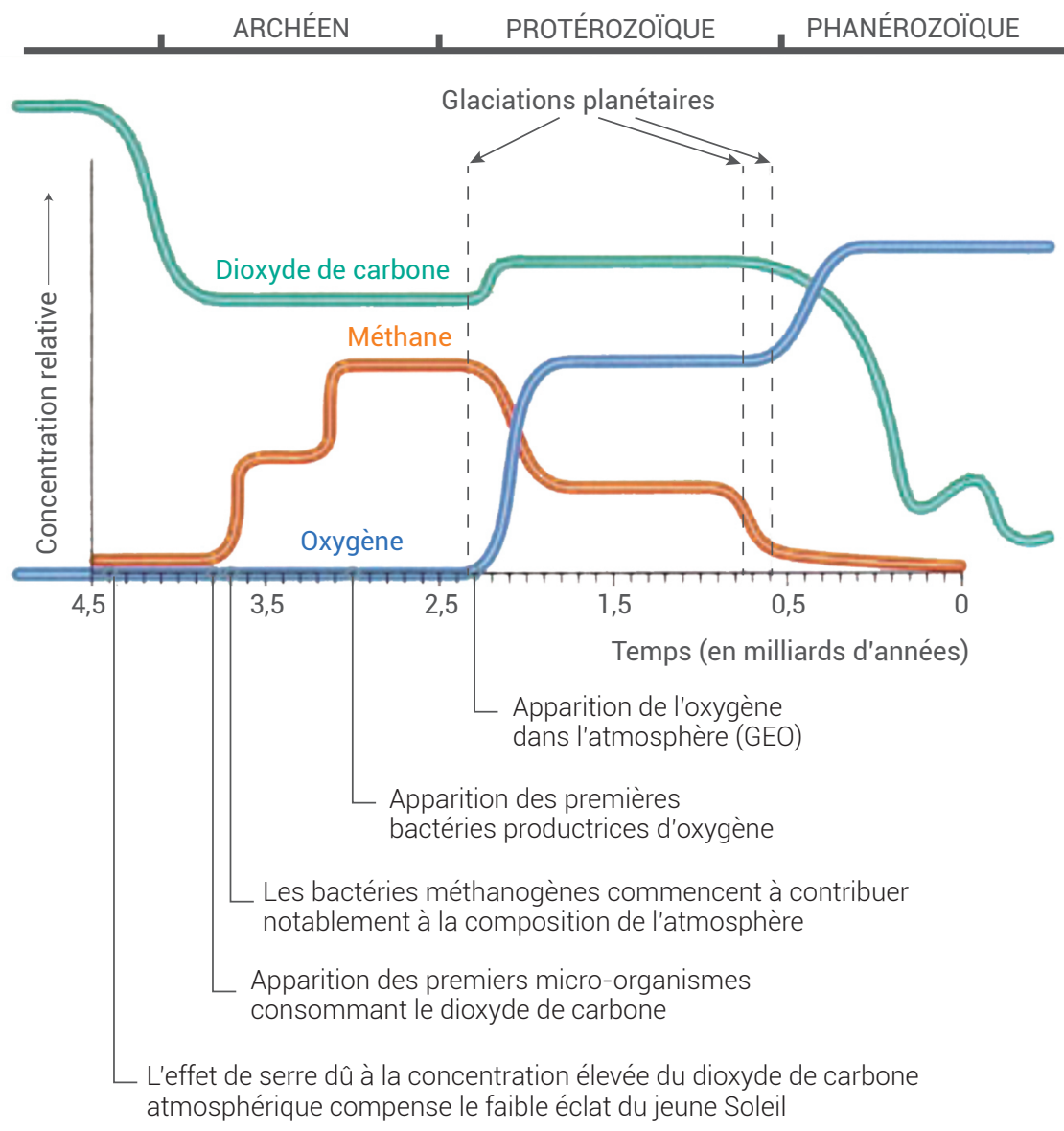

3. Variation de la concentration relative de l'atmosphère terrestre en oxygène, méthane et dioxyde de carbone, de la naissance de la Terre à l'époque présente. À l'échelle des temps géologiques, un marqueur temporel est le Grand Événement d'Oxydation (GEO), entre il y a 2,45 et 2,2 milliards d'années. Cette montée de l'oxygène va conduire à une baisse concomitante du méthane. Mais ce n'est que bien plus tard (pendant le Néoprotérozoïque), entre il y a 800 et 600 millions d'années, que le $\mathrm{CO}_{2}$ va s'effondrer à son tour. (D'après J. Kasting [2]). 
\〉

bassins océaniques jusqu'à il y a environ $1,5 \mathrm{Ga}$, mais au-delà cela reste très difficile. Ceci constitue un mur pour la modélisation des climats. Sans connaissance de la distribution des bassins océaniques et des masses continentales, plus de possibilité de calculer la circulation atmosphérique et océanique. Néanmoins les hypothèses émises par J. Kasting, qui corrèlent l'irruption de l'oxygène avec la première glaciation globale, paraissent tout à fait plausibles. En effet, la montée en puissance de l'oxygène ne modifie pas directement le bilan radiatif de l'atmosphère, car l'oxygène n'est pas un gaz à effet de serre ; par contre, il y a des dommages collatéraux et en particulier chimiques, car l'oxygène va bouleverser la donne dans la distribution des gaz à effet de serre de notre planète. D'une part, il va transformer très rapidement le méthane (qui avait une durée de vie longue dans une atmosphère anoxique) en dioxyde de carbone, qui est un gaz à effet de serre bien moins puissant que le méthane. D'autre part, comme l'oxygène constitue un poison puissant pour les bactéries méthanogènes, celles-ci vont se réfugier dans des niches anoxiques. De fait, l'oxygène va couper la source et augmenter le puits de méthane, et donc bouleverser l'équilibre radiatif de la planète... et c'est le premier accident froid. Pour le second accident, qui est beaucoup plus récent, on dispose d'informations bien plus nombreuses.

\section{Une Terre toute blanche au Néoprotérozoïque}

Cette période de l'histoire de la Terre, qui s'étend d'il y a 750 jusqu'à 620 millions d'années (Ma), est géologiquement très riche d'énigmes et de controverses. Nous allons décrire ici les quatre principaux paradoxes qui ont émergé à la fin du $20^{\mathrm{e}}$ siècle sur cette période. D'abord W. Brian Harland (qui était géologue à l'Université de Cambridge) montrait dès les années 1960 qu'il existait, à cette période, des calottes de glace... au niveau de l'équateur. En effet, sur de très vastes zones, des sédiments glaciaires (des tillites ${ }^{(d)}$ ) mettaient en évidence l'existence d'énormes calottes de glace qui s'étendaient de l'équateur jusqu'aux tropiques. Pour résoudre ce premier paradoxe, un géophysicien australien, George Williams, avait fait l'hypothèse audacieuse que l'obliquité terrestre (angle que fait l'axe des pôles avec le plan de l'écliptique) aurait pu être bien plus élevée au Néoprotérozoïque. En effet, si cette obliquité avait été de l'ordre de $60^{\circ}$, la distribution en latitude des températures annuelles aurait été inversée. C'est-à-dire que la zone la plus froide, en moyenne annuelle, se serait trouvée à l'équateur tandis que les pôles auraient connu des températures plus élevées (fig. 4).

Cette hypothèse était donc très séduisante. Elle expliquait pourquoi à l'époque, avec une forte obliquité terrestre, les calottes

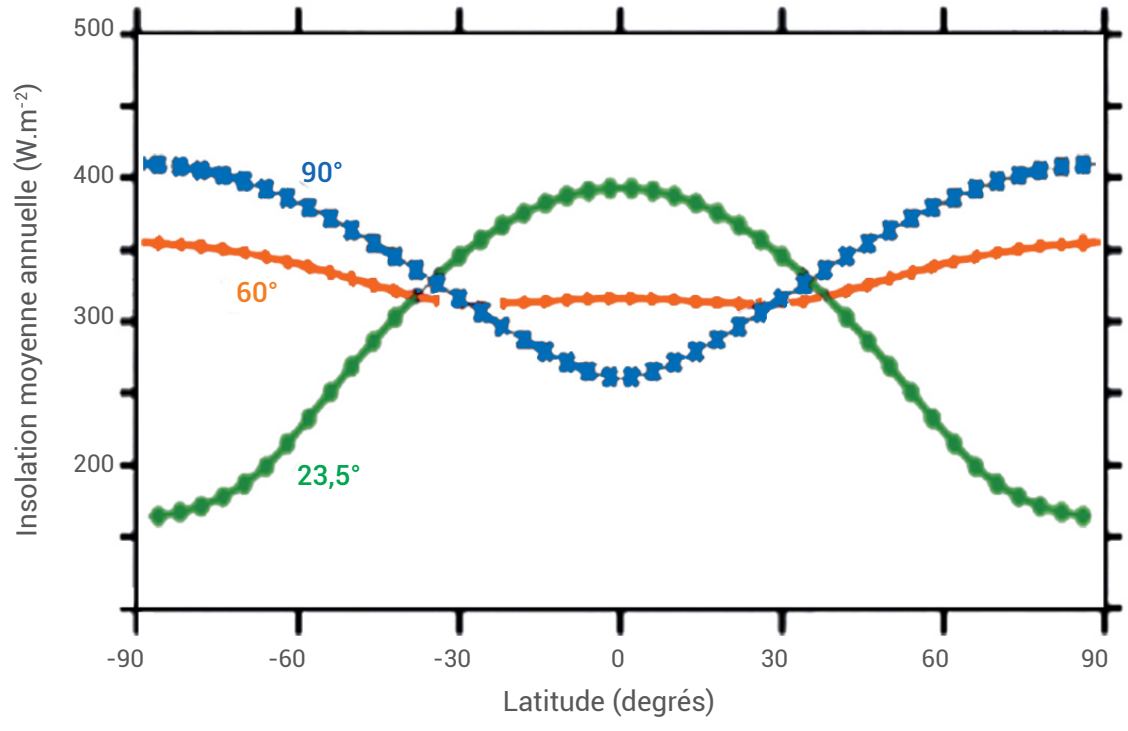

4. Variation de la valeur moyenne de l'insolation terrestre en fonction de la latitude, pour des obliquités terrestres de $23,5^{\circ}$ (l'actuelle), de $60^{\circ}$ et de $90^{\circ}$. s'étaient plutôt développées à l'équateur. Mais elle s'est révélée fausse. D'une part, parce que des astrophysiciens (B. Levrard et J. Laskar [3]) montrèrent qu'on ne disposait d'aucun mécanisme satisfaisant pour faire revenir l'obliquité terrestre à sa valeur actuelle et, d'autre part, parce que Yannick Donnadieu et moi-même démontrâmes qu'avec une telle obliquité les calottes de glace n'auraient pu se développer à cause du très fort cycle saisonnier qui lui aurait été associé.

L'interprétation de Harland, qui observait ces calottes à l'équateur, fut tout naturellement d'imaginer que la Terre s'était complètement englacée au Néoprotérozoïque. Malheureusement, il ne fut pas difficile aux modélisateurs de l'époque de montrer que, si tel avait été le cas, l'albédo, c'està-dire le pouvoir réfléchissant de notre planète, serait passé d'une valeur de 0,3 (valeur actuelle de notre planète bleue) à des valeurs bien plus élevées, de 0,6 à 0,8 (celles d'une planète "boule de neige "). Dans les années 1960, on pensait que seule l'augmentation de la luminosité solaire pouvait avoir permis la déglaciation d'une Terre boule de neige. De ce simple fait, il aurait fallu augmenter la puissance solaire d'un facteur proche de 1,5 pour sortir de cette glaciation globale, et comme vous pouvez le constater sur la figure 2, cela aurait pris des milliards d'années. La glaciation du Néoprotérozoïque aurait donc dû se prolonger au moins jusqu'à nos jours. L'hypothèse de la Terre boule de neige, initialement énoncée par Harland, allait donc faire long feu. Elle fut remisée pendant une petite trentaine d'années.

Trois paradoxes supplémentaires allaient émerger pour la période du Néoprotérozoïque et conduire à l'exhumation de la théorie de la Terre boule de neige.

Le premier paradoxe est la réapparition des formations de fer rubanées (BIF pour Banded Iron Formation). Ces formations, qui se produisent dans un océan sans oxygène, sont très courantes dans l'océan primitif archéen (il y a 3,8 à 2,5 Ga). Elles en constituent même l'archétype. Peu de temps après le GEO, il y a 1,8 milliards d'années, ces formations ont disparu des fonds océaniques. Leur réapparition au Néoprotérozoïque, dans différents bassins océaniques, est donc très surprenante, comme si les fonds marins avaient été de nouveau dépourvus d'oxygène. 
Le second paradoxe tient à l'évolution $\mathrm{du}$ fractionnement entre les isotopes $\mathrm{C}^{12}$ et $\mathrm{C}^{13}$. Celui-ci est marqué par la présence de la vie, qui modifie le rapport de la concentration en $\mathrm{C}^{12}$ par rapport à celle en $\mathrm{C}^{13}$. Or, pour ces périodes du Néoprotérozoïque, on n'observe plus de fractionnement biologique et le taux de $\mathrm{C}^{13}$ dans l'atmosphère reste invariant et égal à celui de sa source volcanique, comme si la biosphère avait été extrêmement réduite.

Enfin, le troisième paradoxe, le plus spectaculaire géologiquement, est lié au fait que les tillites (preuves de glaciations) sont surmontées de montagnes de carbonate (Cap Carbonates) qui se forment aujourd'hui surtout dans les eaux chaudes, comme si après la glaciation le climat avait basculé dans une étuve.

C'est le physicien américain Joe Kirschvink qui fournit une explication à tous ces paradoxes. La Terre avait en effet subi une glaciation globale, comme l'avait suggéré Harland trente ans auparavant. Cependant, la sortie de cette glaciation n'était nullement liée à l'augmentation de la luminosité solaire, mais encore une fois au cycle du carbone. En effet, avec des calottes glaciaires sur les continents et des banquises recouvrant les océans, la Terre n'interagit plus avec le $\mathrm{CO}_{2}$ émis par le volcanisme, car il n'y a plus de contact entre les précipitations et les roches. L'oxygène percole mal dans les océans et la biosphère en prend un coup. Le $\mathrm{CO}_{2}$ s'accumule donc dans l'atmosphère jusqu'à ce que son effet radiatif (comme gaz à effet de serre) soit tel qu'il déglace la Terre gelée. Il ne s'agissait donc plus d'attendre des milliards d'années, mais seulement quelques millions d'années. Le dégel de la planète conduit à former une masse de carbonate pour revenir à l'équilibre.

Cette théorie fut brillamment expliquée et corroborée par Paul Hoffman, de l'université de Harvard, et s'imposa petit à petit dans la communauté scientifique. En effet, elle résolvait la plupart des énigmes.

Mais nous n'avons toujours pas expliqué l'origine de cette glaciation. Pourquoi au Néoprotérozoïque, alors que le Soleil était à 94\% de sa luminosité actuelle et que la Terre semblait sortie d'affaire, au moins pour ce qui est d'échapper à une glaciation, tout à coup la Terre s'englace ? Quelles sont donc les causes de cet accident?

\section{Le tryptique climat/ tectonique/ cycle du carbone}

\section{La dérégulation climatique du Néoprotérozoïque}

$\mathrm{La}$ tectonique, et plus exactement les liens tectonique-climat à travers l'altération des silicates et l'érosion, va jouer un rôle crucial dans un effondrement du $\mathrm{CO}_{2}$ dans l'atmosphére.

Revenons un peu en arrière et prenons de la distance temporelle. Il y a 1,3 à 1,1 Ga, un supercontinent, le Rodinia, s'accrète ; il occupe une position particulière entre les deux tropiques. On a l'habitude, pour simplifier, de dire que la danse des continents sur la lithosphère consiste en la formation d'un supercontinent puis au délitement de ces énormes masses continentales en plaques plus petites. Ce sont les cycles de Wilson, avec des fréquences d'environ 500 millions d'années. C'est ce qui va se produire ici (fig. 5) ; mais ce qui va rendre l'éclatement du Rodinia tout à fait particulier du point de vue climatique, c'est que les petites plaques continentales issues de ce supercontinent vont rester en zone tropicale. Contrairement au Rodinia, plutôt aride au centre et arrosé en périphérie, ces plaques plus petites vont être beaucoup plus intensément arrosées, subir une érosion maximale, et l'altération silicatée va littéralement pomper le $\mathrm{CO}_{2}$ atmosphérique et produire l'englacement.

\〉
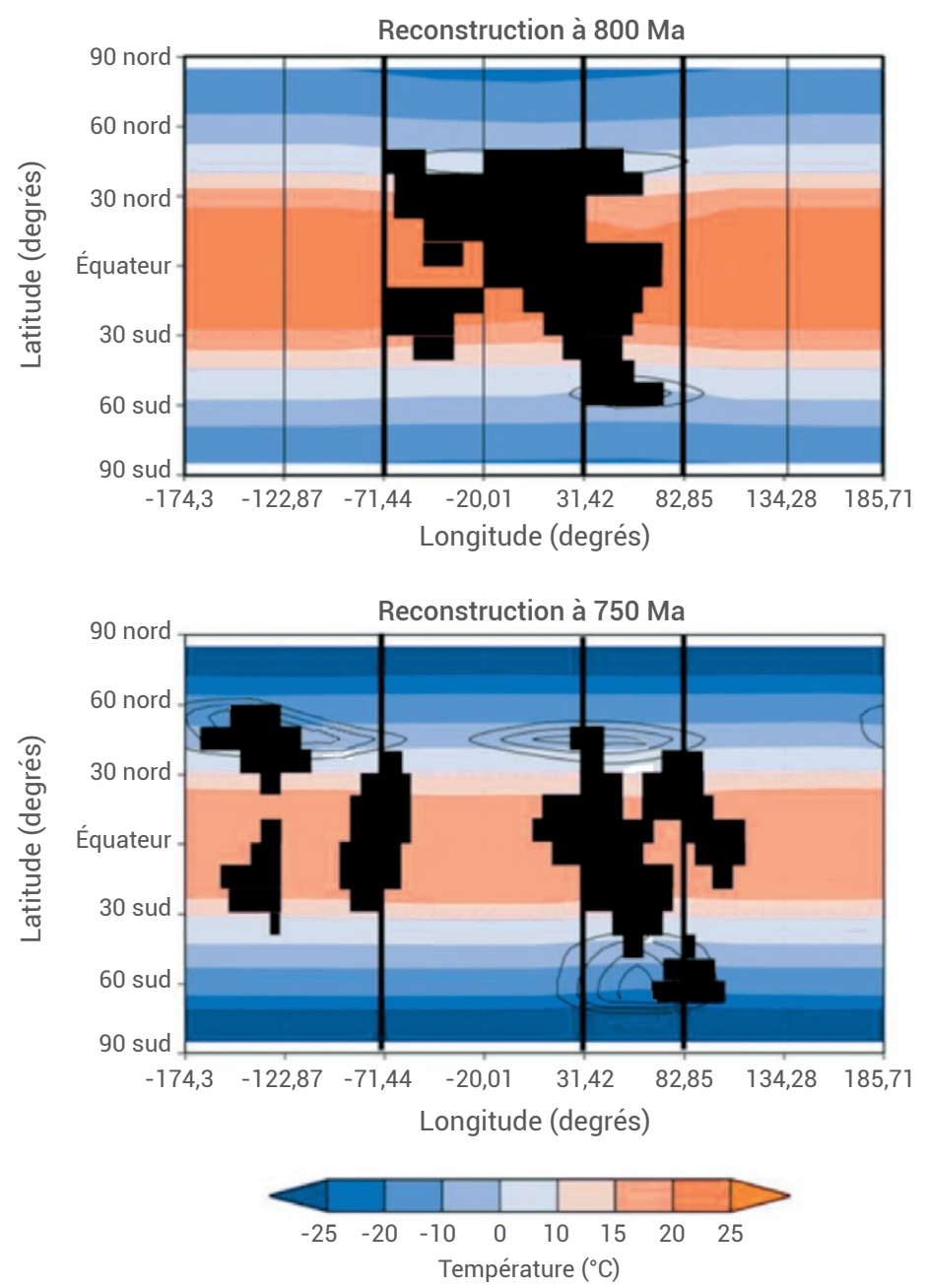

5. Modélisation de l'éclatement du supercontinent Rodinia. Les masques noirs correspondent à la distribution continentale reconstruite à partir du paléomagnétisme. Les températures moyennes à la surface $\left(\mathrm{en}^{\circ} \mathrm{C}\right)$ sont montrées par un code couleur.

(a) Il y a 800 millions d'années, avant la phase glaciaire du Néoprotérozoïque et l'éclatement du Rodinia, le modèle climat-carbone s'équilibre à une concentration atmosphérique en $\mathrm{CO}_{2}$ de 1800 ppm.

(b) Il y a 750 millions d'années, le modèle climat-carbone s'équilibre à une concentration atmosphérique en $\mathrm{CO}_{2}$ de 500 ppm, le Rodinia s'est fragmenté en zone tropicale et les températures ont fortement baissé. (D'après Y. Donnadieu et al. [4]). 
Ce résultat a été simulé à l'aide de modèles couplés climat-carbone grâce à la thèse de Yannick Donnadieu, en collaboration avec Yves Godderis et moi-même [4]. Les résultats en terme de température et de $\mathrm{CO}_{2}$ atmosphérique sont illustrés sur la figure 5 .

Ainsi, si les cycles de Wilson sont nombreux, la configuration géographique de l'éclatement du Rodinia, qui se fait en zone équatoriale, constitue le contexte le plus favorable possible à l'érosion et in fine à l'effondrement du $\mathrm{CO}_{2}$ atmosphérique, et précipite le basculement vers une glaciation globale.

Paul Hoffman [5] a, pendant des années, documenté les deux grandes phases glaciaires du Néoprotérozoïque : d'abord, la glaciation que l'on appelle "sturtienne " et qui intervient entre il y a 720 et $660 \mathrm{Ma}$, puis une seconde phase, la glaciation " marinoenne " (voir la figure de gauche, p. 7), qui est beaucoup plus courte, entre 650 et $635 \mathrm{Ma}$. Le lien entre ces glaciations, la fin du Néoprotérozoïque qui s'ouvre sur l'explosion cambrienne ${ }^{(\mathrm{e})}$ et l'évolution du taux d'oxygène dans l'atmosphère, est encore très mal compris.

\section{Le climat au tempo de la tectonique des plaques}

Quittons ces grandes oscillations climatiques pour nous intéresser au Phanérozoïque, période qui va de l'explosion cambrienne à nos jours (fig. 1b). Sur ces échelles de temps de la dizaine à la centaine de millions d'années, le facteur principal de modulation climatique est la tectonique des plaques. En effet, cette tectonique horizontale (dérive des continents) et verticale (orogénèse) va profondément modifier, à l'échelle de dizaines de millions d'années, les bassins océaniques et donc la circulation des masses d'eau, construire des montagnes qui vont modifier la circulation atmosphérique et le cycle hydrogéologique (mousson).

Cette idée n'est pas nouvelle, Alfred Wegener et Wladimir Peter Köppen ont, peu après la publication de 1924 [6], pensé au fait que la dérive des continents allait changer aussi les climats des continents et leur végétation. Mais ils n'avaient pas perçu le fait que la tectonique des plaques joue non seulement un rôle direct sur le climat, mais également sur le cycle du carbone et sur le taux de $\mathrm{CO}_{2}$ atmosphérique. En effet, si la plupart de la masse continentale se situe dans les tropiques, comme c'était le cas au Néoprotérozoïque, l'érosion silicatée joue à plein et le taux de $\mathrm{CO}_{2}$ dans l'atmosphére diminue, car ce sont des contre, si cette masse se situe près du pôle où le cycle hydrologique est beaucoup plus ténu, il y a peu d'érosion silicatée et le $\mathrm{CO}_{2}$ peut monter dans l'atmosphère.

Cette régulation entre climat et tectonique ne produit, pendant le Phanérozoïque, que très peu de périodes glaciaires. La plupart des périodes géologiques du Primaire et du Secondaire sont chaudes avec de rares exceptions comme au Permo-Carbonifere, il y a 300 millions d'années, où la concomitance d'un continent en position polaire Sud et la relative faiblesse du taux de $\mathrm{CO}_{2}$ conduisent à un englacement important qui dure quelques dizaines de millions d'années. zones de très fortes précipitations. Par

Le Tertiaire (Cénozoïque), une grande descente vers le froid et l'effondrement de la teneur en $\mathrm{CO}_{2}$ atmosphérique

Au cours du Tertiaire, on assiste à une dégradation climatique associée à une baisse du $\mathrm{CO}_{2}$ [7, 8] (fig. 6). La raison de cette baisse prolongée, de 1200 ppm environ à l'Éocène jusqu'à 300 ppm environ au début du Quaternaire, a été associée à l'augmentation de l'érosion silicatée due à la formation de l'Himalaya ou à l'enfouissement rapide du $\mathrm{CO}_{2}$ dans la baie du Bengale [9]. Néanmoins, la cause de cet effondrement du $\mathrm{CO}_{2}$ et sa quantification restent une énigme. Au cours de ce refroidissement, l'Antarctique de l'Est va s'englacer d'abord il y a $34 \mathrm{Ma}$, avec un taux de $\mathrm{CO}_{2}$ de 750 ppm environ. L'Antarctique est en position polaire depuis

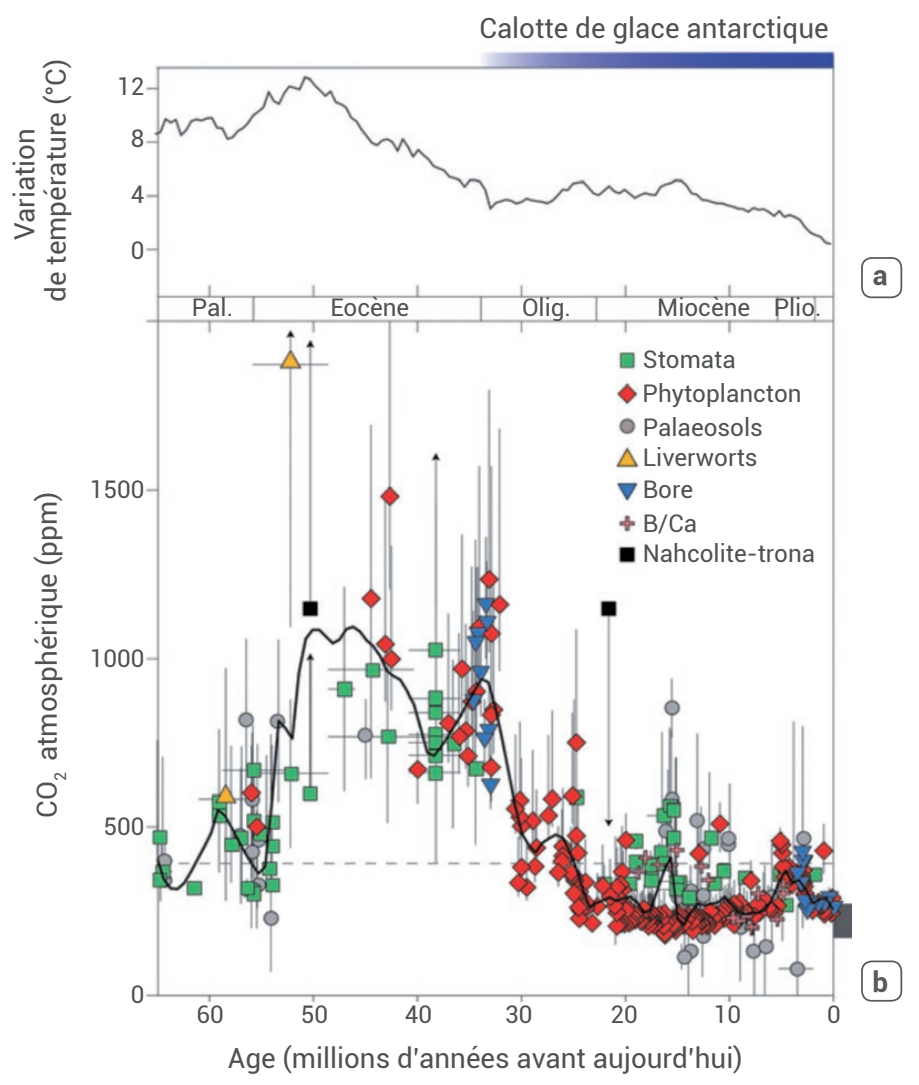

6. L'évolution climatique au cours des 60 derniers millions d'années, depuis le Paléocène jusqu'à l'ère préindustrielle.

(a) Évolution des différences de température globale.

(b) Évolution de la teneur en $\mathrm{CO}_{2}$ atmosphérique. Ces reconstructions sont basées sur différents indicateurs paléoclimatiques (sigles de couleur). (D'après D.J. Beerling et D.L. Royer, Nature Geoscience 4 (2011) 418-420). 
plus de $100 \mathrm{Ma}$, mais ce n'est que lorsque le Passage de Drake s'ouvre, séparant l'Antarctique de l'Amérique du Sud, et surtout lorsque la concentration de $\mathrm{CO}_{2}$ aura suffisamment baissé, que la calotte s'installera. Ce n'est que beaucoup plus tard que l'englacement du Groenland s'effectue pour des valeurs bien plus basses de $\mathrm{CO}_{2}$, 300 ppm environ, il y a 2,7 Ma. Le Groenland, contrairement à l'Antarctique, n'est ni isolé par un courant circumpolaire ni en position polaire. Il faut donc que le $\mathrm{CO}_{2}$ baisse beaucoup plus que pour l'Antarctique pour franchir le seuil radiatif de l'englacement.

On peut, à l'aide de modèles, simuler la croissance de ces calottes de glace qui, une fois formées, sont relativement stables. Quoi qu'il en soit, à l'issue du Tertiaire, il y a $2 \mathrm{Ma}$, la teneur en $\mathrm{CO}_{2}$ s'est effondrée et le Quaternaire s'ouvre sur un monde très froid à l'échelle géologique.

\section{Le Quaternaire}

Depuis l'englacement du Groenland, il y a 2,7 millions d'années, le climat du Quaternaire s'est encore refroidi et de grandes calottes de glace se sont développées périodiquement sur le nord du continent américain et sur le nord de l'Europe. Ces alternances ne sont pas pilotées par la tectonique des plaques, mais par ce que l'on appelle les paramètres orbitaux qui modifient, à des échelles de 10000 à 100000 ans, la quantité d'énergie saisonnière reçue au sommet de l'atmosphère. C'est Milutin Milankovitch [10] qui, pour la première fois, a élaboré une théorie permettant de relier ces variations glaciaires/ interglaciaires aux changements des paramètres orbitaux de notre planète. Ceux-ci sont au nombre de trois. Ils varient périodiquement. Par ordre décroissant de fréquence, ce sont :

- l'excentricité, c'est-à-dire le caractère plus ou moins elliptique de la trajectoire de notre planète autour du Soleil, qui varie avec des fréquences principales de 400000 et 100000 ans ;

- l'obliquité, qui est l'angle que fait l'axe des pôles avec le plan de l'Écliptique (plan dans lequel la Terre décrit sa trajectoire), variant avec une fréquence de 41000 ans ;

- la précession des équinoxes, qui correspond au fait que l'axe de rotation de la Terre décrit un cône, avec une période d'environ 20000 ans.

La prise en compte des variations d'insolation induites par ces paramètres peut faire basculer d'un monde chaud à un monde froid.

De plus, nous savons, grâce aux multiples forages marins, que le climat oscille depuis un million d'années environ, entre des phases glaciaires longues (environ 100000 ans) et des phases interglaciaires courtes (qui peuvent varier entre 10000 et 40000 ans pour les plus longues). Mais, chose plus extraordinaire encore, grâce aux bulles d'air piégées dans les carottes de glace extraites par les forages en Antarctique, nous connaissons avec précision le contenu de l'atmosphère depuis 800000 ans [11]. Ainsi, ces alternances entre phases froides et chaudes sont associées respectivement à des

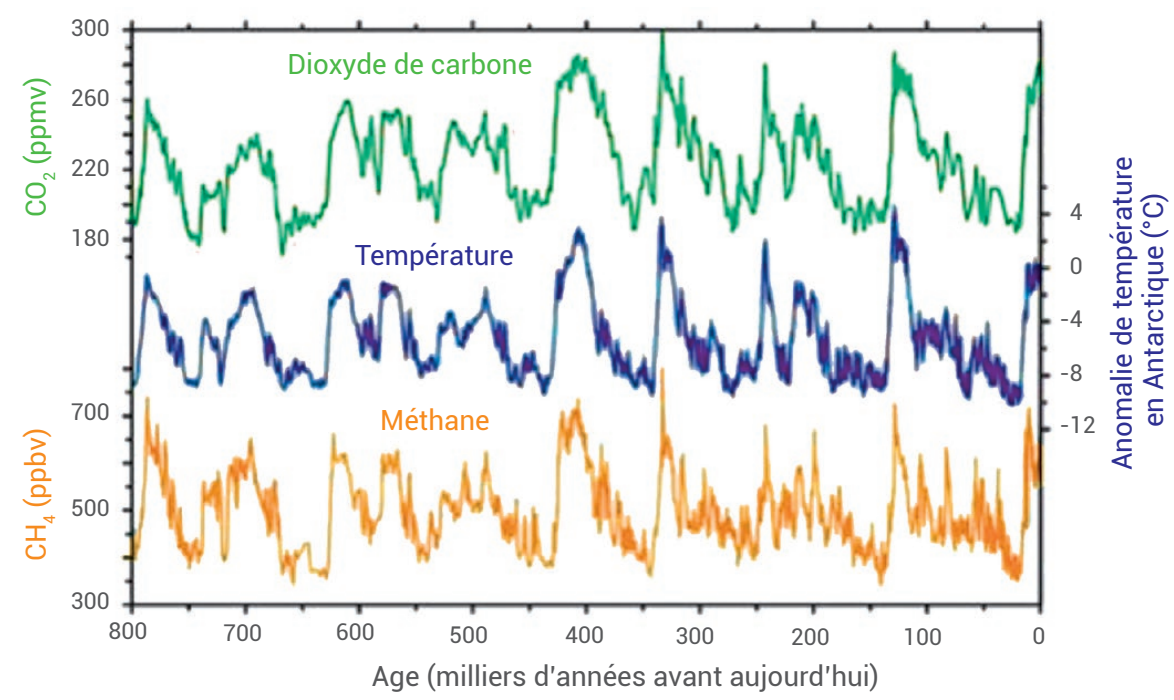

7. Reconstruction des températures et des teneurs en gaz à effet de serre issues des forages en Antarctique (de gauche à droite, du passé vers le présent, en milliers d'années avant 1950). bas niveaux (200 ppm) ou à des hauts niveaux (280 ppm) de $\mathrm{CO}_{2}$ atmosphérique (fig. 7). Le monde glaciaire comporte quatre calottes (voir la figure en couverture de ce numéro) : en plus du Groenland et de l'Antarctique, il y a une énorme calotte, la Laurentide, qui recouvre le nord de l'Amérique et le Canada, et une calotte plus petite, la Fennoscandienne, qui recouvre l'Europe du Nord. Ces deux calottes culminent à environ $3 \mathrm{~km}$ de hauteur et enfoncent sous leur poids le socle rocheux de près d'un kilomètre. Au dernier paroxysme glaciaire, il y a 21000 ans, ces calottes avaient fait baisser le niveau marin d'environ 120 mètres, ce qui modifiait considérablement les contours des continents. En particulier, la Manche n'existait pas, nous pouvions aller à pied sec en Angleterre, ainsi que de l'Australie à la Nouvelle-Guinée. Si ces alternances se déroulent pendant tout le Quaternaire, la mieux connue est la dernière d'entre elles (d'il y a 135000 ans à nos jours). C'est pendant cette période que l'Homo sapiens a conquis l'ensemble de la planète. L'homme a donc évolué dans un monde plutôt froid, avec un très bas niveau de $\mathrm{CO}_{2}$ et la plupart du temps trois calottes de glace dans l'hémisphère nord et une dans l'hémisphère sud, ce qui est très rare à l'échelle géologique. C'est dans ce contexte que va s'effectuer une expérience fulgurante de remise massive de $\mathrm{CO}_{2}$ dans le réservoir atmosphérique, par oxydation des combustibles fossiles.

\section{Le feu d'artifice anthropique}

Aujourd'hui, l'Homme fait parcourir au dioxyde de carbone le chemin inverse de celui du Tertiaire, que nous avons décrit plus haut, et cela non pas en 50 millions d'années, mais en deux siècles, en réinjectant massivement du $\mathrm{CO}_{2}$ dans l'atmosphère (fig. 8). Or, comme nous l'avons vu dans le paragraphe précédent, ce feu d'artifice démarre dans un monde froid où, fait rare dans l'histoire géologique de la Terre, des calottes de glace existent à la surface du globe. Dans ces conditions, le problème de la stabilité des calottes antarctique et groenlandaise se pose. Peuvent-elles physiquement (radiativement) résister aux changements climatiques? Ce problème est d'autant plus préoccupant qu'une remontée du niveau marin de quelques mètres suffirait à poser d'énormes problèmes sur une 
\〉

planète où vivent 7 milliards d'hommes, dont une forte proportion sur les côtes. Bien sûr, il y a une hystérésis liée au changement d'albédo des surfaces enneigées qui conduit au fait que le taux de $\mathrm{CO}_{2}$ pour sortir de la glaciation sera plus élevé que celui qui a permis d'y entrer. Néanmoins, nous avons maintenant dépassé les 400 ppm (fig. 8c) et cette valeur est suffisante pour déstabiliser, à terme, le Groenland qui représente environ $6 \mathrm{~m}$ de niveau marin et l'Antarctique de l'Ouest qui en représente 3 à $5 \mathrm{~m}$. Il faut remonter très loin dans le temps pour savoir quand le taux de $\mathrm{CO}_{2}$ atmosphérique a atteint $400 \mathrm{ppm}$. En effet, grâce aux bulles piégées dans les carottes de glace de l'Antarctique, on a la certitude que depuis près d'un million d'années le taux de $\mathrm{CO}_{2}$ n'a évolué que dans une petite fenêtre de 180 à 280 ppm (fig. 7). Au-delà de ce million d'années, d'autres indicateurs de la teneur en $\mathrm{CO}_{2}$ de l'atmosphère permettent de reconstruire des valeurs équivalentes à celles d'aujourd'hui pour la période du Pliocène moyen, entre il y a 3,3 et 3,0 millions d'années. Quel était alors le climat? Il était plus chaud d'environ $2^{\circ} \mathrm{C}$ que l'actuel, mais le niveau marin lui, était d'environ 10 mètres plus élevé ; en effet, la calotte du Groenland était très réduite et celle de l'Antarctique de l'Ouest également. Dans l'augmentation du niveau des mers, la contribution des calottes glaciaires va devenir le premier acteur devant la dilatation thermique et l'apport des glaciers continentaux. Le dernier rapport du GIEC montre que d'ici la fin du $21^{\mathrm{e}}$ siècle la remontée du niveau marin, simulée par les modèles, peut augmenter de 30 à $90 \mathrm{~cm}$; mais il existe beaucoup d'incertitudes dans ces calculs, nous ne sommes donc pas à l'abri de surprises.

(a) Voir le travail récent très complet de Sébastien Dutreuil dans sa thèse, soutenue le 2 décembre 2016 à l'Université de Paris 1 Panthéon-Sorbonne.

(b) Les cratons sont des parties stables de la lithosphère continentale, vieilles de plus de $2 \mathrm{Ga}$, parvenues à une rigidité telle qu'elles n'ont pu, au cours de l'histoire de la Terre, ni être fragmentées ou déformées, ni subir de mouvement orogénique d'importance.

(c) Les archées sont des micro-organismes unicellulaires de la famille des procaryotes (organismes vivants ne comportant pas de noyau et presque jamais de membrane).

(d) Les titilles sont des roches sédimentaires formées par compaction d'un dépôt fluvio-glaciaire ancien ou par l'accumulation de débris de roches, qui sont entrainées puis abandonnées par les glaciers.

(e) L'explosion cambrienne, qui se situe il y a $540 \mathrm{Ma}$ environ, correspond à l'apparition d'une très grande variété d'espèces végétales et animales, que l'on retrouve dans le registre fossile.
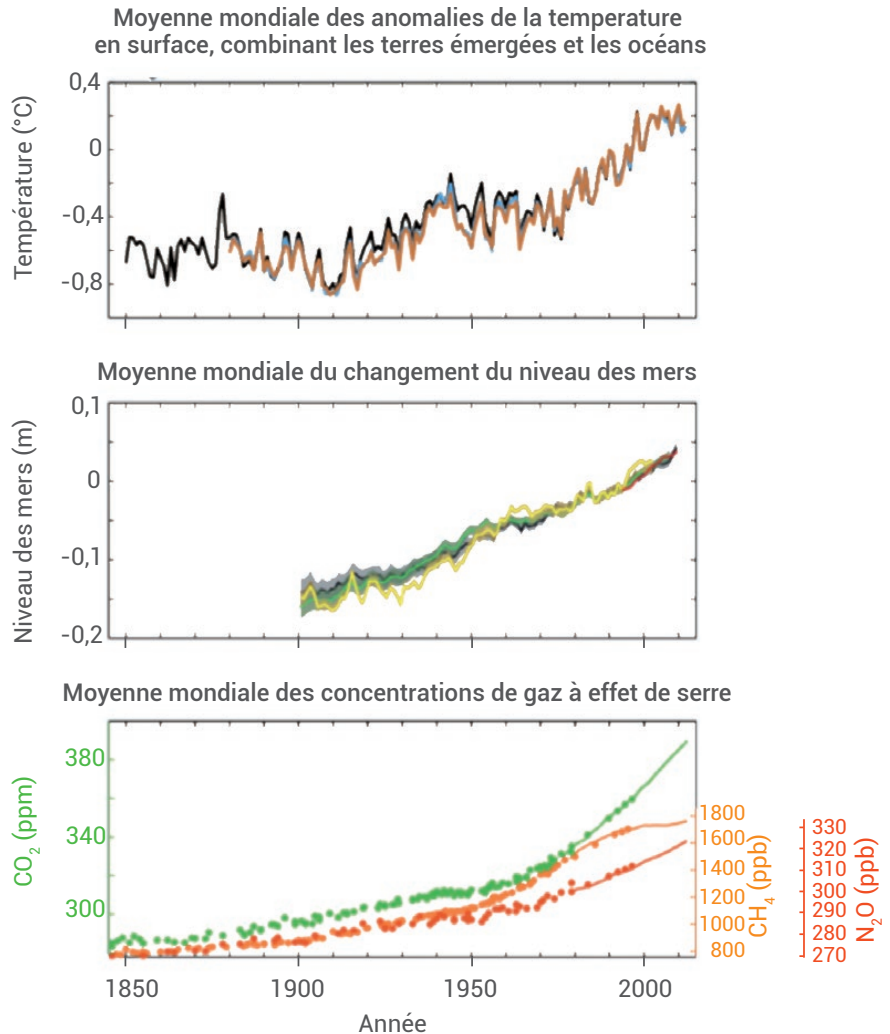

8. Changements climatiques observés depuis l'année 1850. En a et b, les différents ensembles de données sont représentés par des courbes de couleurs différentes.

(a) Moyenne annuelle et mondiale des anomalies de la température de surface combinant les terres émergées et les océans par rapport à la moyenne établie pour la période 1986-2005.

(b) Moyenne annuelle et mondiale de l'évolution du niveau des mers par rapport à la moyenne établie pour la période 1986-2005 pour l'ensemble de données le plus long.

(c) Concentrations atmosphériques des gaz à effet de serre que sont le dioxyde de carbone $\left(\mathrm{CO}_{2}\right.$, vert), le méthane $\left(\mathrm{CH}_{4}\right.$, orange) et l'oxyde nitreux $\left(\mathrm{N}_{2} \mathrm{O}\right.$, rouge), déterminées à partir de l'analyse de carottes de glace (points) et obtenues par mesure directe dans l'atmosphère (courbes). (Source : GIEC, Changements climatiques 2014, Rapport de synthèse, p. 3).

\section{Références}

1. J.E. Lovelock, La Terre est un être vivant. L'hypothèse Gaïa, Éditions du Rocher (1986).

2. J. Kasting, "Méthane et climat ", Pour la Science, 323 (septembre 2004), p. 30.

3. B. Levrard et J. Laskar, "Climate friction and the Earth's obliquity", Geophys. J. Int. 154 (2003) 970-990.

4. Y. Donnadieu et al., "A 'snowball Earth' climate triggered by continental break-up through changes in runoff", Nature 428 (2004) 303-306.

5. P.F. Hoffman et al., "Climate dynamics of Snowball Earth and Cryogenian geologygeobiology", Science Advances (2017).

6. W. Köppen et A. Wegener, Die Klimate der geologischen Vorzeit, Gebrüder Bornträger, Berlin (1924).

7• J.C. Zachos et al., "Trends, rhythms, and aberrations in global climate 65 Ma to present", Science, 292 (2001) 686-693.

8. J.C. Zachos et al., "An early Cenozoic perspective on greenhouse warming and carbon-cycle dynamics" Nature 451 (2008) 279-283.

9. J.C. Duplessy et G. Ramstein, Paléoclimatologie, tome II, chap. 6, EDP Sciences (2013).

10• M. Milankovitch (Belgrade, 1941), Canon of insolation and the ice-age problem, traduit de l'allemand, Israel Program for Scientific Translations and published for the U.S. Department of Commerce and the National Science Foundation (1969).

$11 \cdot$ V. Masson-Delmotte et A. Landais, "Explorer l'évolution du climat grâce aux glaces de l'Antarctique et du Groenland ", Reflets de la physique 41 (2014) 12-17.

\section{Pour en savoir plus}

- A. Knoll, Life on a Young Planet: The first Three Billion Years of Evolution on Earth, Princeton University Press (2003). - J.C. Duplessy et G. Ramstein, Paléoclimatologie, tomes I et II, EDP Sciences (2013).

- G. Ramstein, Voyage à travers les climats de la Terre, Odile Jacob (2015). 